\title{
An evaluation on the performance of two simple triangular bending plate elements
}

\author{
Dian Rahmawati ${ }^{1}$, Imam Jauhari Maknun ${ }^{1}$, and Irwan Katili ${ }^{1, *}$ \\ Universitas Indonesia, Civil Engineering Department, Kampus UI, Depok 16424, Indonesia.
}

\begin{abstract}
This paper will study and compare two different three-node triangular bending plate elements with three degree of freedom per node, i.e. MITC3 and DKMT. Both elements, which were developed based on Reissner-Mindlin plate theory and independent shear strain field, have simple formulation and have already been used widely. In this paper, numerical tests for circular plate case are conducted to verify the performance and show the convergence of these two triangular elements.
\end{abstract}

\section{Introduction}

In structural modelling, the use of triangular element is interesting due to its simplicity and flexibility. Threenode triangular element is mostly used for complex configuration. However, research on triangular is not as much as quadrilateral element. In finite element analysis, many researches show that quadrilateral element have a good performance. As a result, many analyses prefer to use the quadrilateral element [1-10]. This condition should encourage researchers to develop study about triangular element.

The challenge in finite element is how to generate a simple and applicable element formulation to reduce the computational cost, yet still has high accuracy and good convergence.

MITC (Mixed Interpolation of Tensorial Components) concept has been applied for triangular element. MITC3, proposed by Lee and Bathe [11], is one of the popular bending elements. There are quite a lot of studies and developments of MITC3 [12-15]. This threenode triangular element has a simple and general formulation. The behaviour of the element is isotropic, meaning that the stiffness matrix of the element does not depend on the sequence of node numbering.

Previously, Katili [16] proposed simple formulation for triangular bending plate element. The element, called DKMT, is an extension of DKT (Discrete Kirchhoff Triangular) element [17] which is a simple, efficient and reliable element for analysis of thin plates. In the case of thin plate problem, the solution obtained with DKMT element converged to that given by DKT element.

The purpose of this paper is to compare formulation and performance of two triangular elements, i.e. MITC3 and DKMT. Both of them have simple formulation. At the end of this paper, we conduct numerical studies for circular bending plate to compare and evaluate the convergence of both elements.

\section{Formulation of DKMT}

The formulation of DKMT plate element is based on Reissner-Mindlin hypothesis, which only require $\mathrm{C}^{0}$ continuity, and generated by assumed natural strain concept. Transversal shear deformation for this element is expressed with special interpolation called shear projection method. The triangular element has threedegrees of freedom per node (Fig.1).

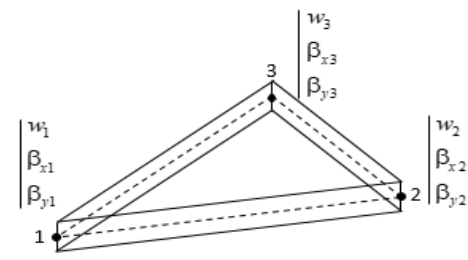

Fig. 1. Triangle element and the degrees of freedom

\subsection{Bending strain matrix}

The DKMT element was first published in 1993 [16]. DKMT element has 3 nodes with 3 degrees of freedom each, which are: $w$ (translation in the $z$ direction), $\beta_{x}$ (rotation in the $z-x$ plane) and $\beta_{y}$ (rotation in the $z-y$ plane). Incomplete quadratic rotation fields for $\beta_{x}$ and $\beta_{y}$ are considered in terms of rotations at the three corners and a temporary variable at mid-side $i-j$ (Fig.2).

$$
\begin{aligned}
& w=\sum_{i=1}^{3} N_{i} w_{i} \\
& \beta_{x}=\sum_{i=1}^{3} N_{i} \beta_{x_{i}}+\sum_{k=4}^{6} P_{k} C_{k} \Delta \beta_{s_{k}}
\end{aligned}
$$

*Corresponding author: irwan.katili@eng.ui.ac.id 
$\beta_{y}=\sum_{i=1}^{3} N_{i} \beta_{y_{i}}+\sum_{k=4}^{6} P_{k} C_{k} \Delta \beta_{s_{k}}$

where: $\quad w_{i}$ is the vertical displacement at node- $i$

$N_{i}$ is the shape function at node- $i$

$\beta_{x_{i}}, \beta_{y_{i}}$ are the rotation in plane of $z-x$ and $z-y$

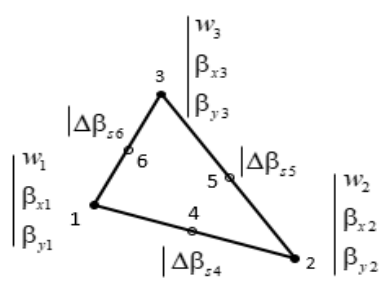

Fig. 2. Kinematic variable of corner node and temporary variable at mid side $i-j$.

$N_{i}$ are the linear shape functions and $P_{k}$ is the quadratic functions:

$N_{1}=\lambda \quad ; \quad N_{2}=\xi \quad ; \quad N_{3}=\eta \quad ; \quad \lambda=1-\xi-\eta$

$P_{4}=4 \lambda \xi \quad ; \quad P_{5}=4 \xi \eta \quad ; \quad P_{6}=4 \lambda \eta$

$C_{k}$ and $S_{k}$ are the direction cosines of side $i-j$ (Fig.2)

$\{\chi\}=\left\{\begin{array}{c}\chi_{x} \\ \chi_{y} \\ \chi_{x y}\end{array}\right\}=\left\{\begin{array}{c}\beta_{x, x} \\ \beta_{y, y} \\ \beta_{x, y}+\beta_{y, x}\end{array}\right\}$

$\beta_{x, x}$ and $\beta_{x, y}$ denote the first derivatives of $\beta_{x}$ with respect to $x$ and $y$, respectively.

The relation between curvature and nodal displacement is expressed in the equation below.

$\{\chi\}=\left[B_{b_{\beta}}\right]\left\{u_{n}\right\}+\left[B_{b_{\Delta \beta}}\right]\left\{\Delta \beta_{s_{n}}\right\}$

where: $\quad\left\{u_{n}\right\}=\left\langle u_{n}\right\rangle^{T}=\left\langle\begin{array}{lllll}\ldots & w_{i} & \beta_{x_{i}} & \beta_{y_{i}} & \ldots\end{array}\right\rangle_{i=1,2,3}^{T}$

$$
\left\{\Delta \beta_{s_{n}}\right\}=\left\langle\Delta \beta_{s_{n}}\right\rangle^{T}=\left\langle\Delta \beta_{s_{4}} \quad \Delta \beta_{s_{5}} \quad \Delta \beta_{s_{6}}\right\rangle^{T}
$$

From eq. (1-4), we obtain the expression of bending strain matrix,

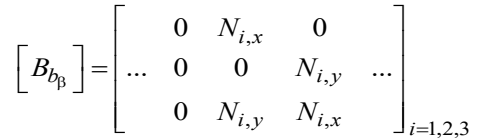

$$
\begin{aligned}
& {\left[B_{b_{\Delta \beta}}\right]=\left[\begin{array}{ccc}
P_{k},{ }_{x} C_{k} \\
\ldots & P_{k}, S_{y} S_{k} & \cdots \\
P_{k},{ }_{y} C_{k}+P_{k},{ }_{x} S_{k} & ]_{k=4,5,6}
\end{array}\right]}
\end{aligned}
$$

$N_{i, x}, P_{k, y}, N_{i, x}$ and $P_{k, y}$ denote the first derivatives of $N_{i}$ and $P_{k}$ with respect to $x$ and $y$, respectively.

\subsection{Shear strain interpolation}

Shear deformation field is approximated linear in each element.

$$
\{\underline{\gamma}\}=\left\{\begin{array}{l}
\underline{\gamma}_{x} \\
\underline{\gamma}_{y}
\end{array}\right\}=\sum_{i=1}^{3} N_{i}\left\{\begin{array}{l}
\underline{\gamma}_{x_{i}} \\
\underline{\gamma}_{y_{i}}
\end{array}\right\}
$$

where: $\underline{\gamma}_{x_{i}}$ and $\underline{\gamma}_{y_{i}}$ are the shear deformations at nodes- $i$

Shear deformation is assumed constant along the edge of element (Fig.3). Shear deformation at node- $i$ is obtained from projection of constant shear deformation $\underline{\gamma}_{s_{k}}$ from the edge of element to element node.

$$
\begin{aligned}
& \left\{\begin{array}{l}
\left\{\underline{\gamma}_{s_{4}}\right. \\
\underline{\gamma}_{s_{6}}
\end{array}\right\}=\left[\begin{array}{ll}
C_{12} & S_{12} \\
C_{31} & S_{31}
\end{array}\right]\left\{\begin{array}{l}
\underline{\gamma}_{x_{1}} \\
\underline{\gamma}_{y_{1}}
\end{array}\right\} ;\left\{\left\{\begin{array}{l}
\underline{\gamma}_{s_{5}} \\
\underline{\gamma}_{s_{4}}
\end{array}\right\}=\left[\begin{array}{ll}
C_{23} & S_{23} \\
C_{12} & S_{12}
\end{array}\right]\left\{\begin{array}{l}
\underline{\gamma}_{x_{2}} \\
\underline{\gamma}_{y_{2}}
\end{array}\right\}\right. \\
& \left\{\begin{array}{l}
\underline{\gamma}_{s_{6}} \\
\underline{\gamma}_{s_{5}}
\end{array}\right\}=\left[\begin{array}{ll}
C_{31} & S_{31} \\
C_{23} & S_{23}
\end{array}\right]\left\{\begin{array}{l}
\underline{\gamma}_{x_{3}} \\
\underline{\gamma}_{y_{3}}
\end{array}\right\}
\end{aligned}
$$

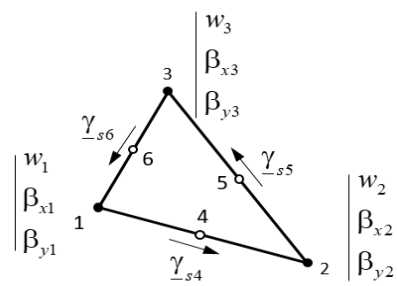

Fig. 3. Constant transverse shear strain along edge $i j$ for DKMT

where:

$$
\begin{aligned}
C_{i j}=\frac{x_{j i}}{L_{i j}} \quad ; \quad S_{i j}=\frac{y_{j i}}{L_{i j}} \quad ; \quad L_{i j}=\sqrt{x_{j i}^{2}+y_{j i}^{2}} \\
x_{j i}=x_{j}-x_{i} \quad ; \quad y_{j i}=y_{j}-y_{i}
\end{aligned}
$$

From eq. (7-9), we obtain:

$\{\underline{\gamma}\}=\left\{\begin{array}{l}\underline{\gamma}_{x} \\ \underline{\gamma}_{y}\end{array}\right\}=\left[B_{s_{\gamma}}\right]\left\{\underline{\gamma}_{s_{n}}\right\}$

with: $\quad\left\{\underline{\gamma}_{s_{n}}\right\}=\left\langle\underline{\gamma}_{s_{n}}\right\rangle^{T}=\left\langle\begin{array}{lll}\underline{\gamma}_{s_{4}} & \underline{\gamma}_{s_{5}} & \underline{\gamma}_{s_{6}}\end{array}\right\rangle^{T}$

$\left[B_{S_{\gamma}}\right]=\left[\begin{array}{lll}\left(\frac{S_{31}}{A_{1}} \lambda-\frac{S_{23}}{A_{2}} \xi\right) & \left(\frac{S_{12}}{A_{2}} \xi-\frac{S_{31}}{A_{3}} \eta\right) & \left(\frac{S_{23}}{A_{3}} \xi-\frac{S_{12}}{A_{1}} \lambda\right) \\ \left(\frac{C_{23}}{A_{2}} \xi-\frac{C_{31}}{A_{1}} \lambda\right) & \left(\frac{C_{31}}{A_{3}} \eta-\frac{C_{12}}{A_{2}} \xi\right) & \left(\frac{C_{12}}{A_{1}} \lambda-\frac{C_{23}}{A_{3}} \eta\right)\end{array}\right](11)$ where:

$A_{1}=C_{12} S_{31}-C_{31} S_{12} ; A_{2}=C_{23} S_{12}-C_{12} S_{23} ; A_{3}=C_{31} S_{23}-C_{23} S_{31}$

Katili [17] proposed the assumed independent transverse shear strain along the side $i-j$ expressed as:

$\underline{\gamma}_{s_{k}}=-\frac{2}{3} \phi_{k} \Delta \beta_{s_{k}} ; \phi_{k}=\frac{2}{\kappa(1-v)}\left(\frac{h^{2}}{L_{i j}^{2}}\right)$

Where $h$ is the thickness of element and $\kappa$ is the shear correction factor (usually $\kappa=5 / 6$ ).

If (12) is applied on all sides of the element, the following matrix relation is obtained:

$\left\{\underline{\gamma}_{s_{n}}\right\}=\left[A_{\phi}\right]\left\{\Delta \beta_{s_{n}}\right\} ;\left[A_{\phi}\right]=-\frac{2}{3}\left[\begin{array}{ccc}\phi_{4} & 0 & 0 \\ 0 & \phi_{5} & 0 \\ 0 & 0 & \phi_{6}\end{array}\right]$

Substituting (13) into (12) we get:

$\{\underline{\gamma}\}=\left\{\begin{array}{l}\underline{\gamma}_{x} \\ \underline{\gamma}_{y}\end{array}\right\}=\left[B_{s_{\gamma}}\right]\left[A_{\phi}\right]\left\{\Delta \beta_{s_{n}}\right\}$

If the assumed shear force and strains are constant on the side, then we obtain:

$\underline{\gamma}_{s_{k}}=\frac{1}{L_{i j}} \int_{0}^{L_{i j}} \gamma_{s} d s=-\frac{2}{3} \phi_{k} \Delta \beta_{s_{k}} ; \gamma_{s}=w, s+\beta_{s}$

Applying (15) on each side- $i j$, we obtain

$\left\{\Delta \beta_{s_{n}}\right\}=\left[A_{\Delta}\right]^{-1}\left[A_{u}\right]\left\{u_{n}\right\} \quad$ where 


$$
\begin{aligned}
{\left[A_{\Delta}\right] } & =-\frac{2}{3}\left[\begin{array}{ccccccc}
\left(1+\phi_{4}\right) & 0 & 0 \\
0 & \left(1+\phi_{5}\right) & 0 \\
0 & 0 & \left(1+\phi_{6}\right)
\end{array}\right] \\
{\left[A_{u}\right] } & =\frac{1}{2}\left[\begin{array}{cccccccccc}
-\frac{2}{L_{12}} & C_{12} & S_{12} & \frac{2}{L_{12}} & C_{12} & S_{12} & 0 & 0 & 0 \\
0 & 0 & 0 & -\frac{2}{L_{23}} & C_{23} & S_{23} & \frac{2}{L_{23}} & C_{23} & S_{23} \\
\frac{2}{L_{31}} & C_{31} & S_{31} & 0 & 0 & 0 & -\frac{2}{L_{31}} & C_{31} & S_{31}
\end{array}\right]
\end{aligned}
$$

Substituting (16) into (4), we define the bending curvatures as:

$$
\begin{aligned}
& \{\chi\}=\left[B_{b}\right]\left\{u_{n}\right\} \\
& {\left[B_{b}\right]=\left[B_{b_{\beta}}\right]+\left[B_{b_{\Delta \beta}}\right]\left[A_{\Delta}\right]^{-1}\left[A_{u}\right]}
\end{aligned}
$$

Introducing (16) into (14) gives:

$$
\begin{aligned}
& \{\underline{\gamma}\}=\left[B_{s}\right]\left\{u_{n}\right\} \\
& {\left[B_{s}\right]=\left[B_{s_{\gamma}}\right]\left[A_{\phi \Delta}\right]\left[A_{u}\right] ;\left[A_{\phi \Delta}\right]=\left[A_{\phi}\right]\left[A_{\Delta}\right]^{-1}}
\end{aligned}
$$

\section{Formulation of MITC3}

\subsection{Bending strain matrix of MITC3}

The displacement function is given as:

$$
w=\sum_{i=1}^{3} N_{i} w_{i} ; \beta_{x}=\sum_{i=1}^{3} N_{i} \beta_{x_{i}} ; \beta_{y}=\sum_{i=1}^{3} N_{i} \beta_{y_{i}}
$$

The relation between curvature and nodal displacement can be expressed in the equation below.

$$
\{\chi\}=\left[B_{b}\right]\left\{u_{n}\right\} ;\left[B_{b}\right]=\left[B_{b_{\beta}}\right]
$$

\subsection{Shear strain interpolation}

The key of MITC3 [11] technique is the use of appropriate assumed strain interpolation and the proper choice of tying points to relate the displacement interpolation and strain interpolation, which is done separately.

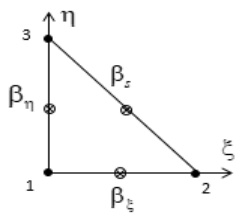

Fig. 4. Tying points

For MITC3, tying points [11-13] are chosen in the mid-points (Fig.4) of sides 1-2, 1-3, and 2-3. Distribution of $\beta_{\xi}$ is assumed constant along $\xi$ direction and $\beta_{\eta}$ is assumed constant along $\eta$ :

$\beta_{\xi}=a_{1}+a_{2} \eta ; \beta_{\eta}=b_{1}+b_{2} \xi ; \beta_{\lambda}=\frac{1}{\sqrt{2}}\left(\beta_{\xi}-\beta_{\eta}\right)$

We obtain the values of $\beta \xi, \beta \eta, \beta \lambda$ at the tying points $A, B$, and $C$.

- At point $A(\eta=0)$, we obtain that $a_{1}=\beta_{\xi(A)}$.

- At point $B(\xi=0)$, we obtain that $b_{1}=\beta_{\eta(B)}$.
- At node $2(\xi=1, \eta=0), \beta_{\lambda}(1,0)=\frac{1}{\sqrt{2}}\left(\beta_{\xi}(1,0)-\beta_{\eta}(1,0)\right)$

- At node $3(\xi=0, \eta=1), \beta_{\lambda}(0,1)=\frac{1}{\sqrt{2}}\left(\beta_{\xi}(0,1)-\beta_{\eta}(0,1)\right)$

- Along edge 3-2, $\beta_{\lambda}(1,0)=\beta_{\lambda}(0,1)$ leading to:

$$
\frac{1}{\sqrt{2}}\left(\beta_{\xi}(1,0)-\beta_{\eta}(1,0)\right)=\frac{1}{\sqrt{2}}\left(\beta_{\xi}(0,1)-\beta_{\eta}(0,1)\right)
$$

Which gives: $a_{2}=-b_{2}=c$

Then the eq. (22) becomes:

$\beta_{\xi}=\beta_{\xi(A)}+c \eta ; \beta_{\eta}=\beta_{\eta(B)}-c \xi$

- At point $C(\xi=1 / 2, \eta=1 / 2)$ :

$\beta_{\lambda(C)}=\frac{1}{\sqrt{2}}\left\{\beta_{\xi(C)}-\beta_{\eta(C)}\right\}$ and

$\beta_{\lambda(C)}=\frac{1}{\sqrt{2}}\left\{\left(\beta_{\xi(A)}+\frac{1}{2} c\right)-\left(\beta_{\eta(B)}-\frac{1}{2} c\right)\right\}$

and we obtain the value of $c$,

$c=\beta_{\eta(B)}-\beta_{\xi(A)}+\beta_{\xi(C)}-\beta_{\eta(C)}$

Substuting eq. (25) into (24), we obtain the expression,

$\beta_{\xi}=\beta_{\xi(A)}+\left(\beta_{\eta(B)}-\beta_{\xi(A)}+\beta_{\xi(C)}-\beta_{\eta(C)}\right) \eta$

$\beta_{\eta}=\beta_{\eta(B)}-\left(\beta_{\eta(B)}-\beta_{\xi(A)}+\beta_{\xi(C)}-\beta_{\eta(C)}\right) \xi$

$\beta_{\xi}$ and $\beta_{\eta}$ at tying points are average value of two points at node.

$\beta_{\xi(A)}=\frac{1}{2}\left(\beta_{\xi_{1}}+\beta_{\xi_{2}}\right) ; \beta_{\eta(B)}=\frac{1}{2}\left(\beta_{\eta_{1}}+\beta_{\eta_{3}}\right)$

$\beta_{\xi(C)}=\frac{1}{2}\left(\beta_{\xi_{2}}+\beta_{\xi_{3}}\right) ; \beta_{\eta(C)}=\frac{1}{2}\left(\beta_{\eta_{2}}+\beta_{\eta_{3}}\right)$

Transverse shear strain field in parametric space:

$\left\{\begin{array}{l}\underline{\gamma}_{\xi} \\ \underline{\gamma}_{\eta}\end{array}\right\}=\left\{\begin{array}{c}w, \xi+\beta_{\xi} \\ w_{\eta}+\beta_{\eta}\end{array}\right\}=\left\{\begin{array}{l}w_{2}-w_{1}+\beta_{\xi} \\ w_{3}-w_{1}+\beta_{\eta}\end{array}\right\}$

Then, from (26-28) we get the expression of shear strain in parametric space:

$\left\{\begin{array}{l}\underline{\gamma}_{\xi} \\ \underline{\gamma}_{\eta}\end{array}\right\}=\left[B_{s_{\xi}}\right]\left\{u_{n}\right\}$

$\left[B_{s_{\xi}}\right]=\frac{1}{2}\left[\begin{array}{rrrrrrrrr}-2 & (1-\eta) & \eta & 2 & 1 & -\eta & 0 & \eta & 0 \\ -2 & \xi & (1-\xi) & 0 & 0 & \xi & 2 & -\xi & 1\end{array}\right]$

Transverse shear strain field in Cartesian system:

$$
\left\{\underline{\gamma}_{\}}\right\}=\left\{\begin{array}{l}
\underline{\gamma}_{x} \\
\underline{\gamma}_{y}
\end{array}\right\}=[j]\left\{\begin{array}{l}
\underline{\gamma}_{\xi} \\
\underline{\gamma}_{\eta}
\end{array}\right\}
$$

Where $[j]$ is the invers of Jacobian matrix.

Introducing (29) into (30), we obtain:

$\{\underline{\gamma}\}=\left\{\underline{\gamma}_{x}\right\}=\left[B_{y}\right]\left\{u_{n}\right\} ;\left[B_{s}\right]=[j]\left[B_{s_{\xi}}\right]$

Mentioned by Jeon et al. in the article [14], MITC3 uses three-points Hammer integration to compute shear stiffness.

\section{Numerical Analysis}

To study the convergence behaviour of MITC3 and DKMT elements, a circular plate problem is analysed. Because of symmetry, only a quarter of circular plate as presents in (Fig.5) is evaluated. Two different boundary 
conditions, i.e. a case of simply supported and a case of clamped supported in perimeter edge, are used with number of element (NELT) of 6, 24, 96, and 384.

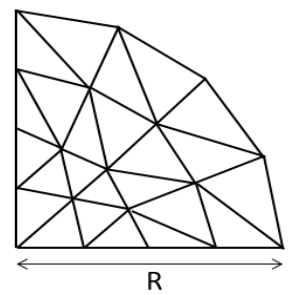

Fig. 5. Circular plate with number of element NELT $=24 . R=$ $5 ; h=0.1,1,2 ; E=10.92$; Poisson's ratio $=0.3$; and uniform load $f_{z}=1$.

Table 1 presents the displacements at the centre of circle $\left(w_{c}\right)$ for soft simply supported case, whereas Table 2 shows the results for clamped boundary condition. Fig. 6 and Fig. 7 show the convergence of $w_{c}$ of both elements.

Table 1. $w_{c}$ for simply supported boundary condition

\begin{tabular}{|c|c|c|c|c|}
\hline $\boldsymbol{R} / \boldsymbol{h}$ & NELT & MITC3 & DKMT & Exact [16] \\
\hline \multirow{4}{*}{50} & 6 & 29056 & 37780 & \multirow{4}{*}{39831} \\
\cline { 2 - 4 } & 24 & 36374 & 39353 & \\
\cline { 2 - 4 } & 96 & 38986 & 39716 & \\
\cline { 2 - 4 } & 384 & 39690 & 39802 & \\
\hline \multirow{4}{*}{5} & 6 & 35.019 & 39.404 & \multirow{4}{*}{41.599} \\
\cline { 2 - 4 } & 24 & 39.705 & 41.038 & \\
\cline { 2 - 4 } & 96 & 41.143 & 41.459 & \\
\cline { 2 - 4 } & 384 & 41.491 & 41.567 & \\
\hline \multirow{4}{*}{2.5} & 6 & 5.044 & 5.554 & \multirow{2}{*}{5.870} \\
\cline { 2 - 4 } & 24 & 5.636 & 5.793 & \\
\cline { 2 - 4 } & 96 & 5.814 & 5.852 & \\
\cline { 2 - 4 } & 384 & 5.857 & 5.866 & \\
\hline
\end{tabular}

Table 2. $w_{c}$ for clamped supported boundary condition

\begin{tabular}{|c|c|c|c|c|}
\hline $\boldsymbol{R} / \boldsymbol{h}$ & NELT & MITC3 & DKMT & Exact [16] \\
\hline \multirow{4}{*}{50} & 6 & 472.3 & 9975.2 & \\
\cline { 2 - 4 } & 24 & 4381.4 & 9991.4 & \multirow{2}{*}{9783.5} \\
\cline { 2 - 4 } & 96 & 8944.1 & 9841.2 & \\
\cline { 2 - 4 } & 384 & 9681.9 & 9796.0 & \\
\hline \multirow{4}{*}{5} & 6 & 6.976 & 11.601 & \multirow{4}{*}{11.551} \\
\cline { 2 - 4 } & 24 & 10.341 & 11.694 & \\
\cline { 2 - 4 } & 96 & 11.271 & 11.587 & \\
\cline { 2 - 4 } & 384 & 11.484 & 11.560 & \\
\hline \multirow{4}{*}{2.5} & 6 & 1.564 & 2.079 & \multirow{2}{*}{2.114} \\
\cline { 2 - 4 } & 24 & 1.969 & 2.127 & \\
\cline { 2 - 4 } & 96 & 2.080 & 2.118 & \\
\cline { 2 - 4 } & 384 & 2.106 & 2.115 & \\
\hline
\end{tabular}
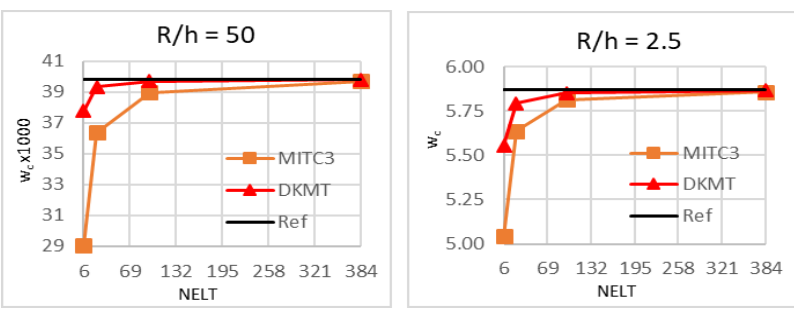

Fig. 6. Convergence of $w_{c}$ for simply supported case
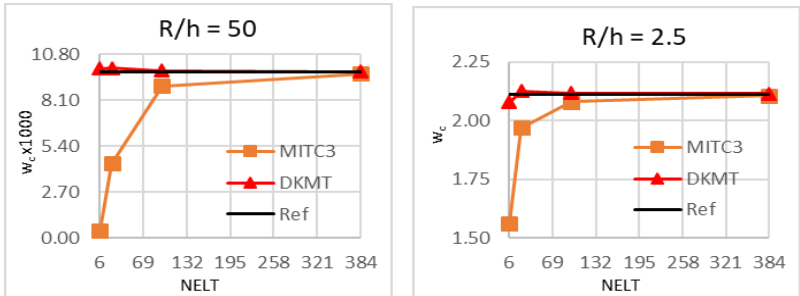

Fig. 7. Convergence of $w_{c}$ for clamped case

\section{Conclusion}

We observe that both elements have good convergence towards the exact solution. DKMT element gives very good results and converges faster than MITC3 element. This is due to the quadratic interpolation functions for the rotations used in DKMT.

The authors gratefully acknowledge financial support from Indonesian Endowment Fund for Education (LPDP).

\section{References}

1. I. Katili, I.J. Maknun, O. Millet, A. Hamdouni, Compos Struct, 132, 166-174 (2015)

2. M. Mahjudin, P. Lardeur, F. Druesne, I. Katili, Struct Saf, 61, 12-21 (2016)

3. F.T. Wong, Erwin, A. Richard, I. Katili, Procedia Eng, 171, 805 - 812 (2017)

4. I. Katili, J.-L. Batoz, I.J. Maknun, A. Hamdouni, O. Millet, Finite Elem Anal Des, 100, 12-27 (2014)

5. I.J. Maknun, I. Katili, O. Millet, A. Hamdouni, Int J Comput Methods Eng Sci Mech, 17, 391-400 (2016)

6. H. Irpanni, I. Katili, I.J. Maknun, Int J Mech Eng and Robot Res, 6, 248-252 (2017)

7. I. Katili, I.J. Maknun, E. Tjahjono, I. Alisyahbana, Int J Tech, 6, 1060-1069 (2017)

8. I. Katili, I.J. Maknun, J.-L. Batoz, A. Ibrahimbegović, https://doi.org/10.1016/j.compstruct.2018.01.043.

9. I. Katili, R. Aristio, Eur J Mech - A/Solids, 67, 92107 (2018)

10. I. Katili, J.-L. Batoz, I.J. Maknun, P. Lardeur, Comput Struct, 204, 48-64 (2018)

11. P.S. Lee, K.J. Bathe, Comput Struct, 82, 945-962 (2004)

12. P.S. Lee, H.C. Noh, K.J. Bathe, Comput Struct, 85, 404-418 (2017)

13. O.P. Gupta (2015), www.researchgate.net/ profile/DrOPGupta/contributions/ May 2015

14. H.M. Jeon, Y. Lee, P.S. Lee, K.J. Bathe, Comput Struct, 146, 91-104 (2015).

15. Y. Lee, K. Yoon, P.S. Lee, Comput Struct, 110-111, 93-106 (2012)

16. I. Katili, IJNME, 36, 1859-1883 (1993)

17. J.-L. Batoz, K.J. Bathe, L.W. Ho, Int J Numer Meth Eng, 15, 1771-1812 (1980) 